\title{
A Novel CSUA Based Data Mining Approach for Mobile Computing Environments
}

\author{
Ashutosh K. Dubey \\ Dept. of Computer Science \& \\ Engineering \\ TITR, Bhopal, India
}

\author{
Ganesh Raj Kushwaha \\ Dept. of Computer Science \& \\ Engineering \\ TITR, Bhopal, India
}

\author{
Jay Prakash \\ Dept. of Computer Science \& \\ Engineering \\ IEM, Mathura, India
}

\begin{abstract}
Data mining services play an important role in the field of Communication industry. Data mining is also called knowledge discovery in several database including mobile databases. In this paper, we discuss and analyze the consumptive behavior based on data mining technology. We discuss and analyze different aspects of data mining techniques and their behavior in mobile devices. We also analyze the better method or rule of data mining services which is more suitable for mobile devices. In this paper, we propose a novel CSUA (Create, Select, Update and Alter) Based data mining approach for mobile computing environments. In CSUA approach, we first propose to create a data set according to the requirement and need then select the data set, then update the data set according to the customers and finally reconstruct the structure of the dataset. It is achieved by CLDC and MIDP component of J2ME.
\end{abstract}

\section{Keywords}

Data Mining, CSUA, CLDC, MIDP.

\section{INTRODUCTION}

The innovations in computer science have made it possible to acquire and store enormous amounts of data digitally in databases, currently giga or terabytes in a single database and even more in the future. Many fields and systems of human activity have become increasingly dependent on collected, stored, and processed information. However, the abundance of the collected data makes it laborious to find essential information in it for a specific purpose. In the late 1980's, the disciplines of knowledge discovery and data mining emerged to help survey the information content of data. It is also use in mobile devices with the use of MIDLET and CLDC component of J2ME. In few years back, mobile extensions to Grid systems have been increasingly proposed in order to support ubiquitous access and selection to the Grid and to include mobile devices as additional Grid resources [1,2].

In today's scenario mobile devices, such as mobile phones, PDAs, notebook and others, provide a basic building block [3][4][5][6].Finding prevalent mobile user patterns and behavior in large amount of data has been one of the major problems in the area of mobile data mining. Particularly, the algorithms of discovering frequent user's behavior patterns in the mobile agent system have been studied extensively in recent years. The key feature in most of these algorithms is that they use a dataset and frequent Item-Sets visited by the customers. In this case, some problems occur because they do not consider that mobile user's behavior patterns are dynamically variable as time passes. In this paper we discuss some of the data mining service which are use in different areas and then apply those services to mobile devices and then apply those DMS services in mobile computing and exploiting the need of DMS in mobile computing environments using CLDC and MIDP components. The Connected, Limited Device Configuration (CLDC) and the Mobile Information Device Profile (MIDP) have emerged as J2ME standards for mobile phone applications development which are used with DMS services. The role of CLDC and MIDP component is to apply Data Mining Services in mobile. Data Mining Services are useful in several sectors including Mobile, WWW, HealthCare scenario etc. We discuss several aspects step-by-step in this paper and analyze those aspects and approaches sequentially. Discuss their advantages and disadvantages and conclude with new concept.

The remaining of this paper is organized as follows. We discuss CLDC and J2Me in Section 2. In Section 3 we discuss about MIDP. The proposed data mining algorithm, namely CSUA in section 4 . In section 5 we discuss about the Challenges. The conclusions and future directions are given in Section 6. Finally references are given in section 7 .

\section{CLDC and J2ME}

The $\mathrm{J} 2 \mathrm{ME}$ architecture is described in general before the components in the J2ME technology are introduced.J2ME applications are also discussed in general, and it is explained how they are made available to end users.J2ME is a highly optimized Java runtime environment. J2ME is aimed at the consumer and embedded devices market. This includes devices such as cellular telephones, Personal Digital Assistants (PDAs) and other small devices. Fig 1 shows the J2ME architecture. Java 2 Standard Edition (J2SE) developers should be familiar with Java Virtual Machines (JVMs) and at least one host operating System (OS).

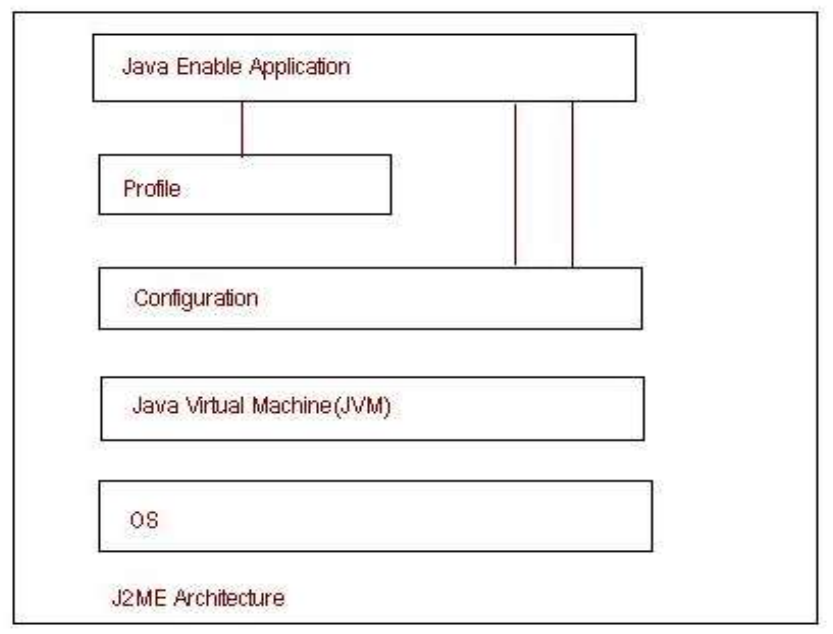

Fig1 J2ME Architecture 
The fundamental branches of the J2ME plstform are configurations. A configuration is a specification that describes a Java Virtual Machine and some set of APIs that are targeted at a specific class of device.

The Connected, Limited Device Configuration is one such specification. The CLDC specifies the APIs for devices with less than $512 \mathrm{~KB}$ of RAM available for the Java system and an intermittent (limited) network connection. It specifies a stripped-down Java virtual machine, called the KVM, as well as several APIs for fundamental application services. Three packages are minimalist versions of the J2SE java.lang, java.io, and java.util packages. A fourth package, javax.microedition.io, implements the Generic Connection Framework, a generalized API for making network connections.

Many J2ME games already exist and enjoy great popularity especially among young generation. Java comes with the immense requirement of the object-oriented programming language for developers to implement new mobile applications [7]. Configurations provide core functionality and a way to provide greater flexibility but no services for managing the application life-cycle, for driving the user interface, for maintaining and updating persistent data on the device or for secure access to information stored on a network server [8].Fig 2 shows the CLDC position in J2ME Architecture.

Several networks have conducted a survey on users' watching behavior [9] which reflects that user behavior pattern recognition is not so easy task; we can achieve this by CLDC and MIDP component. Instead of replacing existing TV service, mobile services should be complementary [10], and offer more interactive means for users to watch their chosen content. The CLDC component specifies the connection between the MIDP profile and the connecting components with the server.

All PDAs are small computing devices that contain an operating system, processor, memory and a port to connect the PDA to peripherals and external computing devices.

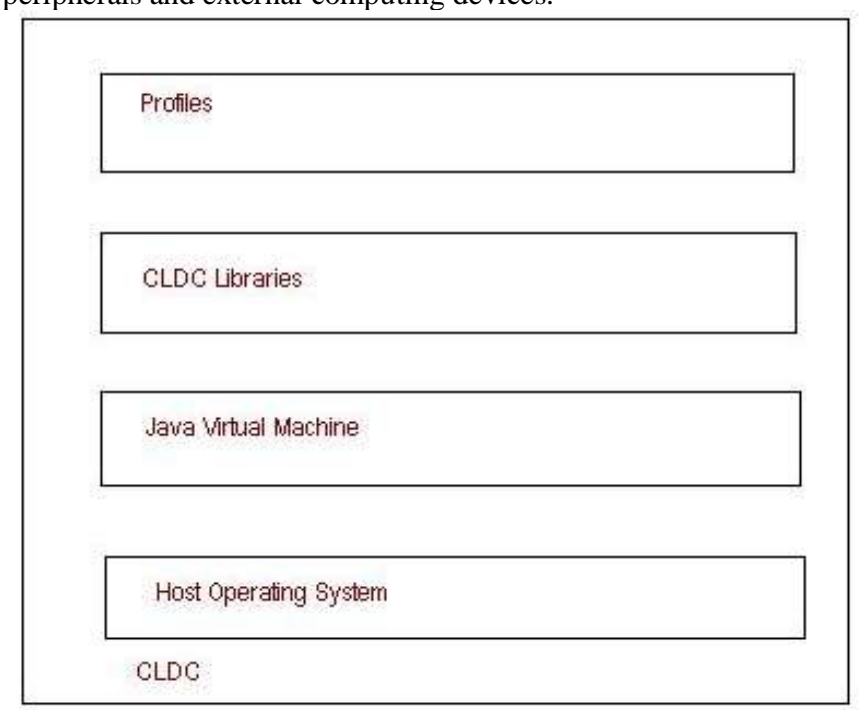

Fig 2 CLDC in J2ME Architecture
The Mobile Information Device Profile is a specification for a J2ME profile. It is layered on top of CLDC and adds APIs for application life cycle, user interface, networking, and persistent storage [11]. An application written for MIDP is called a MIDlet. MIDlet applications are subclasses of the javax.microedition.midlet.MIDlet class that is defined by MIDP.

MIDlets are packaged and distributed as MIDlet suites. A MIDlet suite can contain one or more MIDlets. The MIDlet suite consists of two files:

\section{- Java Application Descriptor (.jad) file}

The Java Application Descriptor file lists the archive file name, the names and class names for each MIDlet in the suite, and other information. This file is used by the mobile device to ensure that device has the minimum requirements to run the application.

- A Java Archive file (.jar) file.

The archive file contains the MIDlet classes and resource files

Java Studio Mobility is an integrated development environment (IDE), based on the Net Beans development platform, that enables you to use J2ME technologies and add special tools that enable you to code and test J2ME applications, such as emulators and obfuscators.

A MIDlet is a J2ME application designed to operate on an small computing device. A MIDlet is defined with at least a single class that is derived from the javax.micoedition.midlet.MIDlet abstract class. The Position of MIDP is shown in Fig 3.

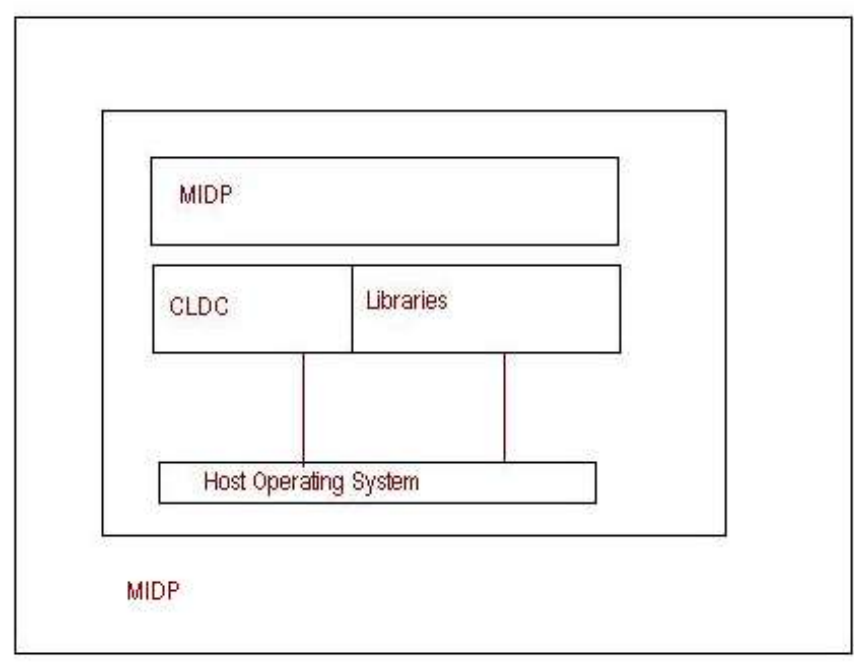

Fig 3 MIDP in J2ME Architecture

\section{Proposed Method: CSUA}

In this section, we describe the proposed method. The entire novel concept is divided into five phases: 1) Create the Data Set according to the user requirement.2) Select the data set when ever needed. 3) Update the data set whenever needed. 4) Alter the data set.5) Mobile communication for the data set.

\section{Algorithm for Creating the Data Set}

\section{MIDP}


For creating the data set we consider the following things:
a) Table size
b) No of Columns
c) No of rows

Assumptions:

Col: Column

Dt: Data Type

V1, v2....vn: Values

Algorithm Create Datasets (CDS)

1. Select the Data Source (WWW, XML, Data Warehouse etc.).

2. Apply the Create table statement on Data Source Create table tablename as select* / Col1, Col2, Col n from data source.

3. Apply the Create table statement[Stand alone] Create table tablename (col1 Dt1, Col2 Dt2.........Col n Dtn) with condition clause.

4. Insert the values considering the domain.

5. For Stand alone Insert into tablename values (v1, v2 .......vn).

6. For step no2 When using universal false condition Insert into table (Select query).

7. Finish.

\section{Algorithm for selecting the Data Set}

For selecting the data set we use select statement

Algorithm Select Datasets (SDS)

1. Select the Data Source (WWW, XML, Data Warehouse etc.).

2. For valuating all columns with rows Select * from datasource.

3. For selected set of columns Select col1, col2,................coln from datasource.

4. For some specified set of conditions Select $* /$ col1, col $2, \ldots \ldots \ldots \ldots \ldots . .$. coln from datasource where condition.

5. Finish.

\section{Algorithm for Updating the Data Set(UDS)}

The update statement is used to change or modify data sets in a database.

1. Select the Data Source (WWW, XML, Data Warehouse etc.) for updating.

2. Update all the rows from a table Update tablename set $\operatorname{col} 1=\mathrm{v} 1, \mathrm{col} 2=\mathrm{v} 2 \ldots \ldots \ldots . \mathrm{coln}=\mathrm{vn}$.

3. Update selected set of rows Update table set $\operatorname{col} 1=\mathrm{v} 1, \operatorname{col} 2=\mathrm{v} 2 \ldots \ldots . \operatorname{coln}=\mathrm{vn}$ where condition.

4. Finish.

\section{Algorithm for Alter the Data Set (ADS)}

By alter statement we can add a column, delete a column and increase and decrease the size of the column in the database.

1. Select the Data Source (WWW, XML, Data Warehouse etc.) for alter.

2. Add new column to the data source.

Alter table tablename add (Col1 Dt1, Col2 Dt2........Coln Dtn).

3. Modify the size of the column If(table is empty)

\{

3a. Increase the size

Alter table tablename modify (Col1 Dt1, Col2

Dt2.........Coln Dtn).

3b. Decrease the size

Alter table tablename modify (Col1 Dt1, Col2 Dt2 ........Coln Dtn).

\}

If(table is not empty)

\{

3c. Increase the size

Alter table tablename modify (Col1 Dt1, Col2 Dt2......... Coln Dtn).

\}

Else

\{

Exit (0);

\}

4. Drop the column

Alter table tablename drop (columns).

5. Finish

\section{Algorithm for Mobile Communication for the Data Set (MCDS)}

1. Select the Data Source (WWW, XML, Data Warehouse etc.) for alter.

2. Apply the MIDP Profile.

3. Using MIDlet establish the connection

4. Apply the packages of J2ME according to the need.

5. Using WTK (Wireless ToolKit) access the result on the simulator.

6. Apply data Mining techniques.

7. Finish.

After analyzing the several aspects of CSUA method the picture is clear for any databases it is easy to manage and whenever necessary we can update the repository system.

We also apply several data mining techniques very smoothly because our data base is consistent because of limiting redundancy in the database. Finally apply the J2ME for mobile devices so that we can coherent the entire above scenario for mobile computing environments.

\section{CHALLENGES}

A number of constraints and technical difficulties faced by researchers, which are discussed in this section. These general problems must be considered for further research in this area to 
propose new technologies for making mobile computing easier. Some of these are:

- The screen size of the mobile is a big limitation. The screen size can affect the approximate visualization of complex results representing the discovered model.

- Mobile navigation facility is also a big task to achieve and implement.

- The overhead due to the communication between MIDLET and Data Mining service should not affect the execution time.

- The experiments on system performance depend almost entirely on the computing power of the server on which data mining task is executed.

We are attempting to implement knowledge discovery applications. Techniques and tools can also be implemented in DMS as decentralized and interoperable services that enable the development of complex system such as distributed knowledge discovery suits.

\section{CONCLUSIONS AND FUTURE DIRECTIONS}

Along with the rapid development of information technology, executing advanced technologies through mobile handset is the prime direction of development. Implementation of intelligent modules on mobile devices through the combination of J2ME and related computing will be the base to introduce data mining features in Mobile Computing. In future we propose a novel data mining algorithm named J2ME-based Mobile Progressive Pattern Mine (J2MPP-Mine) for effective mobile computing. In J2MPP-Mine, we first propose a subset finder strategy named Subset-Finder (S-Finder) to find the possible subsets for prune. Then, we propose a Subset pruner algorithm (SB-Pruner) for determining the frequent pattern. Furthermore, we proposed the novel prediction strategy to determine the superset and remove the subset which generates a less number of sets due to different filtering pruning strategy which is more efficient and less time consuming.

\section{REFERENCES}

[1] M. Migliardi, M. Maheswaran, B. Maniymaran, and P. Mobile Interfaces to Computational, Data, and Service Grid Systems. ACM SIGMOBILE Mobile Computing and Communications Review, 6(4), 2004.

[2] S. Wesner, T. Dimitrakos, and K. Jeffrey. Akogrimo - The Grid goes Mobile. ERCIM, October (59), 2004.

[3] A. Arcelus, M.H. Jones, R. Goubran, and F. Knoefel. Advanced Information Networking and Applications Workshops, 2007, AINAW. 21st International Conference, May 2007.

[4] Raphael M. Bahati and Michael A. Bauer. In ICAS '08: Proceedings of the Fourth International Conference on Autonomic and Autonomous Systems (ICAS'08), pages 88-93, Washington, DC, USA, 2008. IEEE Computer Society.

[5 Michael Beetz, Jan Bandouch, Alexandra Kirsch, Alexis Maldonado, Armin Müuller, and Radu Bogdan Rusu. In Proceedings of the 4th COE Workshop on Human Adaptive Mechatronics (HAM), 2007.

[6] Ralph Bergmann. Ambient intelligence for decision making in fire service organizations. In AmI, pages 73-90, 2007.

[7] Isakow, A. and Shi, H. "Review of J2ME and J2MEbased Mobile Applications", International Journal of Communication and Network Security, Vol. 8 No. 2,pp. 189-198.

[8] Ortiz, 2004a, A Survey of J2ME Today, Sun Developer Network (SDN), viewed 13 August 2007.

[9] http://www.3g.co.uk/PR/Sept2005/1943.htm

[10] http://www.cellular-news.com/story/18707.php

[11] http://docs.sun.com. 